\title{
Über einige Bestandteile der Maiskeime.
}

Von

E. Winterstein und F. Wünsche.

(Aus dem agrikultur-chemischen Laboratorium der Eidgenössizchen Technizchen Hochschule in Zürich.)

(Der Redaktion zugegangen am 6. Oktober 1915.)

Über den Mais liegt eine große Reihe von Arbeiten chemischer Natur vor. Die älteren Arbeiten beschäftigten sich mit der Zusammensetzung der Maispflanze in verschiedenen Entwicklungsstadien vom Beginn der Keimung bis zur Fruchtreife. Trotzdem diese Untersuchungen mit den damaligen unzulänglichen Methoden ausgeführt worden sind, gewähren sie doch einen gewissen Einblick in den Aufbau und die Wanderung der Stoffe, wobei auch auf die Bedeutung der Aschenbestandteile hingewiesen wird.

In unserem Laboratorium sind eine Anzahl von noch nicht publizierten Arbeiten ähnlicher Art ausgeführt worden, unter Zuhilfenahme der neueren Methoden. Diese Arbeiten wurden in der Absicht ausgeführt, einigen Aufschluß über die Bildung der Eiweibstoffe in den Pflanzen zu gewinnen.

Für das Studium dieser Frage schien mit Rücksicht auf die vorliegenden chemischen Untersuchungen die Maispflanze besonders geeignet. Dabei war es angezeigt, über die chemische Zusammensetzung des Embryo des Maiskornes Aufschluß zu erlangen, weshalb sich die vorliegende Arbeit znnächst ausschließlich mit der Untersuchung desselben beschäftigt.

Das zur Untersuchung gelangte Material wurde uns durch Herrn H.C. Humphrey von der Firma "Corn Products Refining 
Company, Newyork» zur Verfügung gestellt. Es sei uns gestattet, Herrn Humphrey und der Corn Products Refining Company auch auf diesem Wege unseren verbindlichsten Dank auszusprechen.

Das Material wurde in zwei Sendungen zugestellt. Die erste Sendung, welche ich mit A bezeichne, bestand aus schon gemahlenen Keimen, während wir bei der zweiten Sendung ganze und nach der gütigen Untersuchung von Professor Dr. C. Schellenberg vollkommen unverletzte Embryonen erhielten. Dieses Material, welches mit B bezeichnet sei, wurde zur quantitativen Analyse und verschiedenen andern Versuchen sorgfältig ausgelesen und von der $4,9 \%$ betragenden Beimengung $(49,49 \mathrm{~g}$ Keime enthielten 2,423 $\mathrm{g}=4,90 \%$ Beimengungen) getrennt. Die Beimengung bestand größtenteils aus Bruchstücken der Chalaza und aus Samenschalen, nur hin und wieder kamen kleine Teilchen von Endosperm vor.

Material A war frisch hergestelit, bei $112^{\circ} \mathrm{F}$. getrocknet und in zugelöteten Blechbüchsen auf schnellstem Wege herbefördert worden, um eine Veränderung tunlichst zu vermeiden.

Zur qualitativen Untersuchung war eine größere Menge extrahierten Materials erforderlich. Die Extraktion großer Mengen des Materials wurde von der Firma Blattmann \& Cie. in Wädensweil in dankenswerter Weise mit Äther und Alkohol nach genauer Vorschrift ausgeführt.

Die bei niedriger Temperatur von den Lösungsmitleln befreiten Extrakte sowie das extrahierte Material wurden uns wieder gut verschlossen zurückgesandt.

Diese Keime können nicht als völlig frei von anhaftenden Beimengungen bezeichnet werden, da sie mit technischen. Mlitteln isoliert und uns in gemahlenem Zustande gesandt worden waren.

$16,84 \mathrm{~kg}$ Ausgangsmaterial gaben bei der Extraktion $8 \mathrm{~kg}$ Ätherextrakt, $2,08 \mathrm{~kg}$ Alkoholextrakt und $6,76 \mathrm{~kg}=\mathrm{ca} .40,1 \%$ entfettetes, mit Alkohol extrahiertes Material.

Material B wurde nach dem Mahlen mit verschiedenen Lüsungsmitteln je nach Bedarf entfeltet, worüber an den betreffonden Stellen näheres mitgeteilt ist. 


\section{Quantitative Untersuchungen.}

1. Trockensubstanzbestimmung.

Das Material A wurde mehrere Wochen im Vakuumexsikkator vorgetrocknet und dann im Wasserstoffstrom bei $105^{\circ}$ bis zum konstanten Gewicht getrocknet. Der Trockensubstanzgehalt betrug $96,22 \%$.

2. Fettbestimmung.

Durch Extraktion mit absolutem Äther und Trocknen des Rückstandes im Wasserstoffstrom. Rohfettgehalt im Material A $53,44 \%$, im Material B 54,20\%. Das Rohfett enthielt 0,01\% N und $0,081 \% \mathrm{P}$, daraus berechnet sich ein Lecithingehalt von $2,11 \%$, für das unentfettete Ausgangsmaterial A 1,14\%.

3. Bestimmung des Phosphors im Alkoholextrakt.

Das mit Äther extrahierte Material wurde 3 mal je $1 / 2$ Stunde mit absolutem Alkohol ausgekocht, abfiltriert, Filtrat vom Alkohol befreit, der Verdampfungsrückstand des Alkohols mit SalpeterSodamischung verbrannt, in verdünnter Salpetersäure gelöst, mit Ammonmolybdat nach W oy gefällt, Phosphormolybdat in Ammoniak gelöst, Lösung mit Magnesiamixtur nach Schmitz gefällt, gab $0,0208 \mathrm{~g} \mathrm{Mg}_{2} \mathrm{P}_{2} \mathrm{O}_{7}=0,02 \% \mathrm{P}$ (im Ausgangsmaterial $29,685 \mathrm{~g})=$ ca. $0,54 \%$ Distearyllecithin.

In den Alkoholextrakt ging also ungefähr gleichviel Phosphor ein, wie in den $\ddot{A}$ therextrakt.

4. Quantitative Bestimmung des Unverseifbaren im Ätherextrakt des Materials A.

$41,545 \mathrm{~g}$ des auf warmem Sandbad im Vakuumexsikkator über Schwefelsäure ca. 4 Stunden getrockneten Ätherextraktes wurden mit $100 \mathrm{ccm}$ absolutem Alkohol erwärmt, hierzu eine Lösung von $8 \mathrm{~g}$ Natrium in $160 \mathrm{ccm}$ absolutem Alkohol gefügt, auf dem Wasserbad erwärmt, Alkohol vertrieben, die entstandenen Seifen bei $80^{\circ}$ getrocknet, fein zerrieben und ca. 12 Stunden mit Äther extrahiert. Naci-Verjagen des Äthers verblieb ein gelblicher, salbenähnlicher Rückstand, welcher mit wenig Alkohol und ca. 700 .cm Wasser versetzt, milchige 
Trübung gab; nach dem Absitzen, Filtrieren und Trocknen wurden so $0,750 \mathrm{~g}=1,81 \%$ des Ätherextraktes als Unverseifbares erhalten. Beim Versuch der Umkrystallisation löste sich nicht alles im $90 \%$ igen Alkohol, es verblieb eine wachsartige, rötlichgelbe Masse, welche löslich in Äther und in Chloroform war und $0,149 \mathrm{~g}=0,36 \%$ betrug, während $0,601 \mathrm{~g}=1,45 \%$ in verdünntem Alkohol löslich waren.

\section{Aschenbestimmung.}

4,804 g Trockensubstanz gaben $0,065 \mathrm{~g}=1,37 \%$ Asche.

6. Stickstoff bestimmungen.

a) Gesamtstickstoff in der mit Äther entfetteten mit Alkohol extrahierten Substanz A nach Kjeldahl ergab in der lufttrockenen Substanz (mit 9,76\% Feuchtigkeit) 4,13\%, auf die Trockensubstanz umgerechnet $4,47 \%$ Gesamtstickstoff.

b) Eiweißstickstoff nach Stutzer mil Kupferhydroxyd bestimmt $4,26 \%$.

c) Basenstickst off einschlieBlich Ammoniakstickstoff 0,10\%.

Daraus berechnet sich folgende Verteilung des Stickstoffs auf die entfettete mit Alkohol extrahierte, wasserfreie Trockensubstanz.

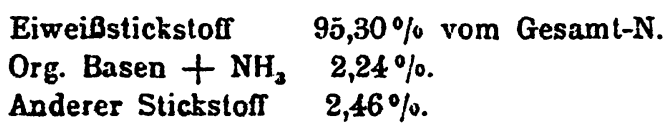

In dem nur mit Äther extrahierten Material wurden folgende Stickstoffmengen gefunden :

\section{Auf entfettete}

Substanz

$3,93 \%$ Eimeil-N

$0,32 \%$ Basen-N

$0,46 \%$ \% Anderer $\mathrm{N}$
Auf das Ausgangsmaterial $1,81 \%$

$0,15 \%$

$0,21 \%$
Voin Gesaml-N

83,44

6,79

9,77

1,71\% Gesamtstickstoff.

Eine Untersuchung der sorgfältig von allen Beimengungen getrennten Embryonen (Material B) hatte folgendes Ergebnis:

94,95\% Trockensubstanz.

$2,00 \%$ Gesamtstickstoff $=4,61 \%$ auf entfelteto Kcime berechnet.

- $56,69 \%$ Ätherextrakt. 


$\begin{array}{ll}\text { Gesamtstickstoff ') } & 5,00 \% \\ \begin{array}{l}\text { Durch Kupferbydroxyd fäll- } \\ \text { bar nach Stutzer }\end{array} & 4,41 \%=88,2 \% \text { vom Gesamt-N } \\ \text { Durch Phosphorwolfram- } & \\ \text { säure (ällbar } & 0,49 \%=9,8 \% \text {, , } \\ \text { Anderer Stickstoff } & 0,10 \%=2,0 \% \text {, , }\end{array}$

7. Lösliche Kohlenhydrate.

2,3295 g der mit Äther extrahierten Trockensubstanz des Materials A wurden einige Stunden mit Wasser von $40-50^{\circ}$ digeriert und hierauf abfiltriert. Der Rückstand wog getrocknet 2,1446 g. Die Trockensubstanz enthielt demnach 0,1849 $\mathrm{g}$ $=7,93 \%$ Wasserlösliches. Das Filtrat wurde zur Entfernung der Eiweißkörper mit Bleiessig versetzt, solange noch eine Trübung entstand, und auf $500 \mathrm{ccm}$ aufgefüllt. $50 \mathrm{ccm}$ der überstehenden, klaren Flüssigkeit gaben beim Kochen mit Allihnscher Lösung nur unwägbare Spuren von reduziertem Kupfer. Auch nach dreistündigem Kochen von $250 \mathrm{ccm}$ der klaren Flüssigkeit mit 5\% iger Schwefelsäure wurde die Allihnsche Lösung nur in Spuren reduziert, so daß nachweisbare Mengen von wasserlöslichen Kohlenhydraten nicht vorhanden sind.

\section{Untersuchung auf Pentosen.}

$50 \mathrm{~g}$ lufttrockene, ausgelesene Keime des Materials B $=47,55 \mathrm{~g}$ Trockensubștanz wurden auf $85-90^{\circ} \mathrm{erwärmt}$, mit $180 \mathrm{ccm}$ Wasser von $85^{\circ}$ digeriert, nach 10 Minuten abgepreßt und $3 \mathrm{mal}$ mit heißem Wasser ausgewaschen. Der Preßsaft wurde nochmals filtriert, abgesaugt und vom überstehenden Fett abgelassen. Das Filtrat von $274 \mathrm{ccm}$ war etwas trüb, zeigte saure Reaktion und gab im Gegensatz zur Autolysenflüssigkeit (s. S. 330) mit Phloroglucin keine Reaktion auf Pentose, jedoch mit Mohlisch-Reagenz den violetten Ring.

Die Hälfte des Filtrates wurde mit $59 \mathrm{ccm}$ konzentrierter Salzsäure versetzt und zur Furfuroldestillation verwendet. Die einzelnen Destillate betrugen je $30 \mathrm{ccm}$, das achte der Destillate gab mit Anilinacetatpapier keine Realstion mehr. Die Pentosen wurden nach Tollens bestimmt.

1) Die Zahlen beziehen sich auf ausgelesene und entfettete Keime vom Material $B$. 
Insgesamt wurde erhalten : $0,0246 \mathrm{~g}$ Phloroglucid $=0,030 \mathrm{~g}$ Pentose $=$ ca. $0,13 \%$ Pentose, während bei der Autolyse ca. $0,85 \%$ gefunden worden waren (s. S. 330). Die Natur der Pentose ist nicht bestimmt worden.

Man darf also wohl vermuten, daß die vorhandenen pentosehaltigen Komplexe bei der Autolyse gespalten werden, und daß diese Spaltung höchstwahrscheinlich die Pentoside betriff, da im Pflanzenreiche Pentosane spaltende Fermente unbekannt sind.

II. Qualitative Untersuchungen.

Wie aus der quantitativen Untersuchung hervorging, enthalten die von uns untersuchten Embryonen auffallend wenig nichteiweißartiger Stickstoffverbindungen, und die Darstellung dieser Substanzen war im vorliegenden Falle mit erheblichen Schwierigkeiten verknüpft.

\section{A. Voruntersuchung des Fettes.}

a) Unverseifbares.

Es wurden $50 \mathrm{~g}$ Ätherextrakt des Materials A in $200 \mathrm{ccm}$ absolutem Alkohol gelöst, hierauf mit einer Lösung ron $8 \mathrm{~g}$ Natrium in $250 \mathrm{ccm}$ absolutem Alkohol verseift, zur Trockne eingedunstet und fein zerrieben.

Zur Gewinnung ron event. rorhandenem Phytosterin wurde das erhaltene Pulver im Soxhlet-Apparat 6 Stunden mit wasserfreiem Äther extrahiert, der Ätherextrakt eingedunstet und mit kochendem Alkohol aufgenommen. Beim Abkühlen entstand eine amorphe Ausscheidung, wahrscheinlich verhin-• derten Spuren von Glycerin und Seifen eine Krystallisation. Daher wurde die alkoholische Lösung in Wasser gegossen, die entstandene Fällung abfiltriert und aus kochendem absoluten Alkohol umkrystallisiert. Es resultierten reinweiße, glänzende, schmale Blättchen vom Schmelzpunkt 135-136 .

Eine Probe davon, gelöst in Chloroform, gab mil Schwefelsäure (spez. Gew. 1,47) die Hessesche Reaktion; eine zweite, in Chloroform gelöste Probe wurde mit 3 Tropfen Essigsäure- 
anhydrid, hierauf mit 5 Tropfen konzentrierter Schwefelsäure versetzt, gab rosa Färbung, welche allmählich in dunkelrotviolett überging.

Aus den angegebenen Versuchen und dem konstatierten Schmelzpunkt darf geschlossen werden, daß die erhaltenen Krystalle Sitosterin sind, was auch noch aus den auf S. 317 angeführten Untersuchungen der Benzoylverbindung bestätigt wird.

b) Fettsäuren.

Der Rückstand von der Ätherextraktion wurde in kochendem Wasser gelöst, mit Schwefelsäure angesäuert, mit Kochsalz ausgesalzen.

Das erhaltene klare Öl der Fettsäuren wurde im Scheidetrichter mit Wasser ausgewaschen, wobei sich nach einiger Zeit die höheren Fettsäuren krystallinisch ausschieden.

\section{B. Untersuchung des Rohfettes.}

a) Unverseifbares.

$1000 \mathrm{~g}$ Ätherextrakt des Materials A wurden im großen Rundkolben mit 1/2 I Alkohol am Rückfluß aufgekocht und dann eine Mischung von $1 \mathrm{l}$ Alkohol mit $320 \mathrm{~g} \mathrm{NaOH}$ gelöst in möglichst wenig Wasser, zugefügt, wobei eine heftige Reaktion eintrat. Nach 6 stündigem Kochen der entstandenen Seifen am Rückfluß wurde der Alkohol mit überhitztem Wasserdampf vertrieben. Die anfangs übergehenden Dämpfe rochen stark nach Pyridin und Trimethylamin; sie wurden in verdünnter Schwefelsäure aufgefangen.

Nach möglichst vollständigem Vertreiben des Alkohols wurden die verbliebenen Seifen (ca. $2620 \mathrm{~g}$ ) in 4 flache Schalen verteilt und je $80 \mathrm{ccm}$ konzentrierte Salzsäure und $80 \mathrm{ccm}$ Wasser sowie je $190 \mathrm{~g}$ Kochsalz zugefügt.

Unter ständigem Rühren wurde nun das Wasser vertrieben und der Rückstand im Wasserbad bei $95^{\circ}$ getrocknet.

Die Seifen wurden in 2 Portionen rnit Äther je 15-20 Stunden extrahiert, die hellgelbe ätherische Lösung im Scheidetrichter von noch vorhandenem. Wasser getrennt. Nach dem 
Abdestillieren des Äthers verblieb eine schaumig aufgeblasene dunkelgelbe, krystallinische Masse. Ein Teil derselben wurde in wenig Alkohol gelöst, die Lösung in Wasser gegossen, wobei jedoch eine milchähnliche, nicht filtrierbare Emulsion resultierte. Diese wurde ausgeäthert und nach Vereinigung mit dem Ganzen im Kohlensäurestrom von Äther und Wasser befreit, der Rückstand nochmals mit wasserfreiem Äther aufgenommen, die ätherische Lösung zur Entfernung von anhaftenden Seifen filtriert und nach dem Vertreiben des Äthers gewogen, Ausbeute 19,43 g

\section{$=$ ca. $1,95 \%$ Unverseifbares im Rohfett.}

Einige weitere Versuche zur Reinigung des Phytosterins durch Auflösen in Alkohol und Ausfällen in destilliertem Wasser fielen wiederum erfolglos aus. Die opalescierende wüsserige Lösung reagierte schwach sauer. Anscheinend waren noch Spuren von Fettsäuren vorhanden, daher wurde zur Trennung von diesen das .Unverseifbare, in ca. $500 \mathrm{ccm}$ absolutem Alkohol gelöst und mit alkoholischer Natronlauge erwärmt. Die Lösung färbte sich dabei dunkelrotbraun, es fiel ein weißer Niederschlag anorganischer Natur sowie ein gelber, fettiger Satz (löslich in Wasser) aus, wovon abfiltriert wurde. Der nach dem Abdestillieren des Alkohols verbliebene rotbraune Rückstand war flüssig bei Wasserbadtemperatur, erstarrle beim Abkühlen. Er wurde mit Äther extrahiert, die hellgelbe ätherische Lösung, vom Äther befreit, gab 9,34 g Rohphytosterin.

Das Rohphytosterin wurde durcb Umkrystallisieren aus Alkohol und Äther gereinigt. Schmelzpunkt $136^{\circ}$.

Hiervon worden $2,48 \mathrm{~g}$ mit $4,2 \mathrm{~g}$ Benzoylchlorid auf dem Ölbad bei $160^{\circ}$ \& Minulen erhitzt (die Lösung bezw. Reaktion war schon bei $110-120^{\circ}$ eingetrelen), dann wurde langsam abgekühlt, wobei die Schmelze zu einer grünlichbraunen Nasse erstarrte.

Das überschüssige Benzoylchlorid wurde durch Kochen mit Wasser zu Benzoesäure hydrolysiert, welche durch wiederholtes Auskochen mit Wasser und Filtrieren entfernt wurde. Es' verblieb ein hellgelber, grobkörniger Rückstand, welcher 
mit wenig absolutem Alkohol aufgekocht, nach dem Erkalten filtriert und mit Alkohol gewaschen, dann aus Äther umkrystallisiert wurde. Schmelzp. $144,5^{\circ}$ korr. Ausbeute $0,96 \mathrm{~g}$. Der Schmelzpunkt des Sitosterylbenzoats ist $145,5^{\circ}$.

Es liegt also Sitosterin vor.

b) Fettsäuren im Rohfett.

Eine quantitative Trennung der einzelnen Fettsäuren wurde nicht ausgeführt. Um jedoch einen ungefähren Anhaltspunkt über die Anwesenheit von niederen Fettsäuren zu erhalten, wurde folgendes Verfahren eingeschlagen:

Die Hälfte der nach der Extraktion mit Äther verbliebenen Natronseifen (entsprechend $500 \mathrm{~g}$ Fett) wurde in ca. 81 Wasser von $70^{\circ}$ gelöst und die Fettsäuren mit Schwefelsäure in Freiheit gesetzt.

Die wässerige Lösung wurde von den überstehenden festen Fettsäuren abgelassen und dreimal mit Äther ausgeschüttelt. Die ätherische Lösung wurde mit Wasser gewaschen und mit wasserfreiem $\mathrm{Na}_{2} \mathrm{SO}_{4}$ entwässert; sie gab nach dem Vertreiben des Äthers $14,48 \mathrm{~g}$ niedere wasser- und ätherlösliche Fettsäuren, ca. $=2,90 \%$. Die Untersuchung des Waschwassers sowie der mit Äther ausgeschüttelten wässerigen Lösung ist weiter unten beschrieben.

Die im Wasser unlöslichen Fettsäuren wurden in Äther gelöst, zuerst mit konzentrierter Sodalösung schwach alkalisch gemacht, hierauf mit verdünnter Schwefelsäure schwach angesäuert und im Scheidetrichter mit destilliertem Wasser gewaschen, bis das Waschwasser frei von Schwefelsäure und von Salzsäure ablief. Das Waschwasser wurde mit dem oben erwähnten vereinigt.

Die ätherische Lösung gab nach dem Trocknen mit wasserfreiem Natriumsulfat und Vertreiben des Äthers

$397,66 \mathrm{~g}$ höhere Fettsäuren $=79,55 \%$.

Die vereinigten Waschwasser wurden filtriert und mit überhitztem Wasserdampf die flüchtigen Fettsäuren übergetrieben. Das Destillat wurde mit $22 \mathrm{ccm}{ }^{n} / 5$ Barytwasser neu- 
tralisiert, eingeengt und das Baryum mit Schwefelsäure entfernt. Beim Verestern der verbliebenen Fettsäuren mit Äthylalkohol trat Ananasgeruch auf; daher wurde die geringe Menge als Buttersäure in Rechnung gestellt, woraus sich

berechnet.

$$
0,38 \mathrm{~g}=0,08 \% \text { Buttersäure }
$$

Die oben zuerst erwähnte wässerige Lösung von ca. 10 l, welche mittels Äther von sniederen Fettsäuren * ausgeschüttelt worden war, hatte hellgelbe, etwas grünliche Färbung. Sie wurde mit Natronlauge schwach alkalisch gemacht, stark eingeengt, nach dem Ansäuern mit Schwefelsäure von den ausgeschiedenen Salzen abfiltriert und aus dem Filtrat die flüchtigen Fettsäuren mit Wasserdampf übergetrieben.

Das Destillat verbrauchte zur Neutralisation mit Phenolphthalein als Indikator $100 \mathrm{ccm}$ n/s Barytwasser, was auf Essigsäure berechnet $1,2 \mathrm{~g}=0,24 \%$ ergibt.

Aus den Untersuchungen des Rohfettes resultiert nun:

$\begin{array}{lr}\text { Sitosterin } & 1,94 \%, \\ \text { höhere Fettsäuren } & 79,55 \%, \\ \text { niedere } & 2,90 \%, \\ \text { Buttersäure } & 0,08 \%, \\ \text { Essigsäure } & 0,24 \% .\end{array}$

C. Phosphatidgewinnung.

Es wurden ca. $51 / 2 \mathrm{~kg}$ Embryonen $B$ gemahlen und ca. 14 Tage im Perkolator mit Äther durch Diffusion extrahiert, der Rückstand zerrieben und mit Äther so lange gewaschen, bis der Extrakt eine schwache Phosphorreaktion zu geben begann. Es wurde dadurch verhindert, daß die Phosphatide in den Äther eingingen; eine Abscheidung der Phosphatide aus sehr fettreichen Lösungen ist fast unmöglich.

Der an der Luft getrocknete Rückstand wurde nun zweimal mit 95\% igem Alkohol ausgekocht und abgepreßt. Die alkoholische Lösung, auf dem Wasserbad eingeengt und im Exsikkator über $\mathrm{H}_{z} \mathrm{SO}_{4}$ stehen gelassen, gab nach zwei Wochen keine Krystallisation vonevent. vorhandener Raffinose oder Rohrzucker. Der alkoholische Extrakt wurde nun 
einigemal mil Äther verrührt, bis die ätherische Lösung farblos war, wozu ca. 41 Äther verwendet wurden.

Die ätherische Lösung wurde in kleinen Portionen mit ca. 21 Wasser, dem $1 \mathrm{ccm}$ konzentrierter Salzsäure zugesetzt war, vorsichtig durch langsames Schwenken ausgeschüttelt; es entstand dabei keine Emulsion. Nach dem Trocknen mit wasserfreiem Natriumsulfat wurde die ätherische Lösung auf dem Wasserbade vom Äther befreit, der erhaltene Sirup in wenig kaltem Äther gelöst und in viel auf $20^{\circ}$ abgekühltes Aceton gegossen, wobei eine Fällung entstand, von welcher dekantiert und filtriert wurde. Die Fällung wurde nochmals in Äther gelöst und mit Aceton gefällt. Die so erhaltene Fällung löste sich nur zum Teil in Petroläther.

Der in Petroläther unlösliche Anteil löste sich wiederum nur teilweise in Äther. Die ätherische Lösung gab nach dem Verjagen des Äthers 5,1 g eines Produktes mit 2,53\% P und 2,41\% Zucker (berechnet als Glukose):

0,1870 g Substanz, verbrannt mit Salpeter-Sodamischung, Lösung gefällt nach Woy mit Ammonmolybdat, hierauf nach Schmitz mit Magnesiamixtur, gab 0,0170 g $\mathrm{Mg}_{2} \mathrm{P}_{2} \mathrm{O}_{7}$ $=2,53 \% \mathrm{P}$.

Die Substanz gab nach einstündiger Hydrolyse nur Spuren von Reduktion der Fehlingschen Lösung. 3,310 g gaben nach fünfstündigem Kochen mit $5 \%$ iger Schwefelsäure $0,0138 \mathrm{~g} \mathrm{CuO}$ $=0,0080 \mathrm{~g}$ oder 2,41\% Zucker (berechnet als Glukose).

Die Petrolätherlösung wurde wieder in stark mit Eis gekühltes Aceton gegossen, wobei eine Fällung entstand, die getrocknet $36,2 \mathrm{~g}=$ ca. $0,66 \%$ des Materials B ausmacht. Dieses Phosphatid enthielt $2,87 \% \mathrm{P}$ und $3,91 \% \mathrm{~N}$.

$$
\begin{gathered}
0,9168 \mathrm{~g} \text { gaben } 0,0924 \mathrm{~g} \mathrm{Mg}_{2} \mathrm{P}_{2} \mathrm{O}_{7}=2,81 \% \mathrm{P} \\
0,9026 \mathrm{~g} \text { gaben } 0,0950 \mathrm{~g} \mathrm{Mg}_{2} \mathrm{P}_{2} \mathrm{O}_{7}=2,93 \% \mathrm{P} . \\
\text { Mittel } 2,87 \% \mathrm{P} .
\end{gathered}
$$

$1,0124 \mathrm{~g}$ gaben nach Kjeldahl verbrannt $0,03775 \mathrm{~g}=3,73 \% \mathrm{~N}$ $0,5525 \mathrm{~g}$ gaben $0,02262 \mathrm{~g} \mathrm{~N}=4,09 \% \mathrm{~N}$.

Mittel 3,91\% N.

Das so erhaltene Phosphatid wurde nach dem von G. Trier ${ }^{1}$ )

1) Diese Zeitschr., Bd. 78 (1912) S. 496. 
angegebenen Verfahren auf Colamin (Aminoäthylalkohol) untersucht :

$28,2 \mathrm{~g}$ wurden durch Kochen mit $300 \mathrm{ccm} \mathrm{21/2} \%$ iger Schwefelsäure hydrolysiert, nach dem Abkühlen von den Fettsäuren abfiltriert, diese in Äther gelöst und die Ätherlösung wiederholt mit Wasser ausgeschüttelt, dem etwas $\mathrm{H}_{2} \mathrm{SO}_{4}$ beigefügt war. Filtrat und Waschwasser wurden auf dem Wasserbade eingeengt, vereinigt, mit reinem Baryt alkalisch gemacht und ron den Baryumsalzen befreit. Das eingeengte Filtrat wurde von der kleinen Menge ausgeschiedener Baryumsalze abfiltriert und die Lösung mit Bleiessig versetzt, solange noch ein Niederschlag auftrat, welcher abfiltriert und ausgewaschen wurde. Das Filtrat wurde mittels $\mathrm{H}_{2} \mathrm{~S}$ entbleit, die Lösung zur Trockne eingedunstet, der Rückstand durch wiederholtes Eindunsten mit Alkohol und $\mathrm{HCl}$ von der Essigsäure, hierauf im Vakuum über Natronkalk von überschüssiger $\mathrm{HCl}$ befreit.

Die verbliebenen Salze wurden mit absolutem Alkohol extrahiert, das Filtrat des alkoholischen Extraktes eingedunstet, der Rückstand abermals extrahiert und die filtrierte alkoholische Lösung mit alkoholischer Sublimatlösung gefällt. Der Quecksilberniederschlag wurde aus Wasser umkrystallisiert, mit $\mathrm{H}_{2} \mathrm{~S}$ zerlegt und das Cholin als Platinsalz identifiziert:

$0,2769 \mathrm{~g}$ enthielten $0,0874 \mathrm{~g}=31,56 \% \mathrm{Pt}$, für $\left(\mathrm{C}_{3} \mathrm{H}_{14} \mathrm{ONCl}\right)_{2} \mathrm{PtCl}_{4}$ berechnet sich $31,68 \% \mathrm{Pt}$.

Das Filtrat von der Quecksilberfällung wurde vom Alkohol befreit, gab braunen Rückstand, welcher in heißem Wasser schwer löslich war, wobei ein kleiner schwarzer Rückstand verblieb. Die Lösung wurde mittels $\mathrm{H}_{9} \mathrm{~S}$ von $\mathrm{Hg}$ befreil, das Filtrat einigemal mit Wasser vorsichtig eingedunstet (zur Entfernung von $\mathrm{HCl}$ ), der Rückstand hierauf getrocknet und mit Alkohol aufgenommen, wobei geringer, flockiger Niederschlag verblieb, von welchem abfiltriert wurde. Die alkoholische Lijsung gab mit alkoholischer Platinchloridlösung einen geringen Niederschlag, welcher abfiltriert wurde. Das Filtrat wurde mit $\mathrm{H}_{2} \mathrm{~S}$ von $\mathrm{Pt}$ befreit, vorsichtig eingedunstet, gab beim Stehen über $\mathrm{H}_{2} \mathrm{SO}_{4}$ nach dem Impfen mit salzsaurem Colamin keine Ausscheidung. Der Sirup wurde daher mit Salzsäure 
(spez. Gew. 1,1) und konzentrierter Goldchloridlösung versetzt, nach längerem Stehen bildete sich eine krystallinische Ausscheidung und anscheinend etwas reduziertes Gold. Es wurde daher von der Ausscheidung abfiltriert, das Filtrat mittels $\mathrm{H}_{2} \mathrm{~S}$ vom Gold befreil, eingeengt zum Sirup, durch wiederholtes vorsichtiges Eindunsten mit Wasser die Salzsäure entfernt, mil Alkohol aufgenommen, zum Sirup eingedunstet, mit einem Krystall von Colaminchlorhydrat geimpft und im Exsikkator über $\mathrm{H}_{2} \mathrm{SO}_{4}$ stehen gelassen, nach 14 Tagen keine Krystallausscheidung.

Die Lösung gab mit Phosphorwolframsäure weiße, mit Phosphormolybdänsäure gelbe, käsige Ausscheidung, KaliumWismutjodid rotorange, NeBlers Reagens weiße Trübung, Natriumpikrat ölige, Pikrolonsäure braune Flocken, Fehlingsche Lösung negativ.

Die mittels Goldchlorid erhaltene Krystallmasse (s. o.) wurde mit heißem Wasser behandelt, vom Gold abfiltriert, mit etwas Salzsäure und Goldchlorid versetzt, gab beim Abkühlen eine kleine Menge braunen Niederschlags, von welchem abfiltriert wurde; das Filtrat wurde eingeengt, mit etwas Salzsäure versetzt, gab nach dem Stehen im Exsikkator über Schwefelsäure dunkelorange, zusammenhängende Krystalle, welche bei $157^{\circ}$ korr. sintern (unter schwachem Aufschäumen) und bei $184^{\circ}$ (korr.) schmelzen.

$0,1422 \mathrm{~g}$ verloren beim Erhitzen auf $100^{\circ} 0,0013 \mathrm{~g}=0,42 \% \mathrm{H}_{2} \mathrm{O}$, 0,1409 getrocknetes Goldsalz gaben $0,0691 \mathrm{~g} \mathrm{Au}$ und 0,2004 $\mathrm{g} \mathrm{AgCl}$ $=49,05 \% \mathrm{Au}$ und $35,15 \% \mathrm{Cl}$,

berechnet für Colaminchloraurat $\mathrm{C}_{2} \mathrm{H}_{8} \mathrm{ON} \mathrm{AuCl} \mathrm{Cl}_{4} 49,17 \% \mathrm{Au}$ und $35,36 \% \mathrm{Cl}$.

Somit ist das Vorhandensein von Cholin und Colamin im Phosphatid von Maisembryonen erwiesen.

D. Untersuchung auf Inositphosphorsäure.

$21 / 2$ Kilo des lufttrocknen, mit Alkohol und Äther extrahierten Materials wurden mit großen Menger-Wasser von $30^{\circ}$ únter Zusatz von verdünnter Säure längere Zeit digeriert und die Lösung vom Rückstand durch Abfiltrieren und Abpressen 
gewonnen und aus dieser Lösung die inositphosphorsauren Salze nach bekanntem Verfahren isoliert.1) Die erhaltene Fällung wurde mit Alkohol digeriert und der abgesogene Rückstand bei $80^{\circ}$ getrocknet. Die Ausbeute betrug $15,4 \mathrm{~g}$ $=0,25 \%$. Es repräsentierte eine weiße amorphe kreidige Masse, welche sich in verdünnter Essigsäure vollständig auflöste, diese Lösung gab beim Erhitzen eine weiße Ausscheidung, die beim Erkalten wieder verschwand. Beim Erhitzen mit starker Schwefelsäure bei $158-162^{\circ}$ wurde Inosit erhalten. Die Ausbeute davon war bei weitem nicht quantitativ. ${ }^{2}$ )

\section{E. Darstellung der Eiweißsubstanzen.}

1. Wasserlösliche Eiweißstoffe.

Die von den inositphosphorsauren Salzen befreite Lösung wurde mil Gerbsäure unter Zusatz von einigen Kubikzentimetern Bleiessig gefällt, die gut ausgewaschene Fällung wurde mit Baryt verrieben, das vom Baryumtannat getrennte Filtrat wurde mit Kohlendioxỵd gesättigt und die Lösung vorsichtig eingedunstet. Es wurde eine amorphe, schwach gefärbte Substanz erhalten, welche die bekannte Farbenreaktion auf Eiweißkörper gab. Die Menge dieser wasserlöslichen, durch Hitze nicht koagulierbaren Eiweißsubstanz betrug 4,6 $\mathrm{g}=0,08 \%$.

\section{Globuline.}

Durch Extraktion mit 10\%iger Kochsalzlösung wurden kleine Mengen von Globulinen erhalten. Die Ausbeute daran betrug $0,02 \%$.

3. Alkalilösliche Eiweißstoffe.

139. g entfetteter Trockensubstanz wurden mit $0,2 \%$ iger Natronlauge digeriert, das Filtrat mit verdünnter Essigsäure ausgefaillt und die sorgfältig ausgewaschene Fällung mit Alkohol und Älher getrorknet. Ausbeute 162,4 $\mathrm{g}=11,65 \%$, berechnet auf nicht entfettete Substanz $4,8 \%$.

3) Siehe Bircls:m. Arbeitsmethoden v. Abderhalden Bd. 2, S. 268.

; Vfl. II. S. Posternak, Compl. rend. Bd. 37, S. 202. 
$100 \mathrm{~g}$ von dieser Eiweißsubstanz wurden nach der Methode von E. Fischer auf Aminosäuren untersucht. Es wurden Alanin, Prolin, Leucin und Phenylalanin aufgefunden. Bei der Untersuchung der IV. Esterfraktion, welche in einer Ausbeute von $14,4 \mathrm{~g}$ erhalten wurde und bei $16-19 \mathrm{~mm}$ bei $102^{\circ}$ überdestillierte, wurde eine Beobachtung gemacht, die darauf hindeutet, daß diese Eiweißsubstanz höchstwahrscheinlich eine besondere Aminosäure enthält.

Diese Fraktion wurde mit Äther vom Phenylalanin befreit; die wässerige Lösung wurde mit Baryt verseift, da keine Ausscheidung von asparaginsaurem Kupfer stattfand, wurde die Lösung vom Baryt mit Schwefelsäure befreit; beim Eindunsten unter vermindertem Druck wurden 6,4 g Aminosäuren erhalten. Diese wurde in 4 Krystall-Fraktionen zerlegt. Fraktion 1 gab etwas Leucin, ebenso Fraktion 2; Isoleucin wurde nicht aufgefunden.

3. Krystallfraktion war etwas dunkler gelb, sie wurde aus Wasser umkrystallisiert und gab kugelige, nierenförmige Aggregate, deren Schmelzpunkt $266^{\circ}$ im geschlossenen Rohr war. Sie gab ein Kupfersalz wie die 2. Fraktion.

4. Krystallfraktion. Diese bestand aus reinweißen Krystallen von anscheinend einheitlicher Zusammensetzung. Sie wurde in wenig heißem Wasser gelöst, die Lösung reagierte sauer und wurde mit aufgẹschlämmtem Kupferhydroxyd versetzt, solange sich dieses noch löste. Die dunkelblaue Lösung gab beim Kochen spontane Ausscheidung eines dicken Krystallbreies. Das ausgefallene Kupfersalz löste sich nur äußerst schwer in viel kochendem Wasser. Die heiße Lösung aus den letzten Anteilen des Krystallbreies war ganz blaßblau, beim Abkühlen krystallisierte noch eine kleine Menge aus, welche, abgesaugt und getrocknet, eine hellblaue verfilzte Masse mit starkem Seidenglanz gab. Das aus Wasser umkrystallisierte Kupfersalz zersetzte sich bei $230^{\circ}$ unter starkem Aufschäumen. Es bestand aus hellblauen, zu Büscheln und Garben vereinigten mikroskopisch feinen Nädelchen. Dieses Kupfersalz war trotz seiner geringen Löslichkeit äußerst hygroskopisch, es gab im Vakuum über Schwefelsäure nur einen Teil des Wassers ab, 
enthielt auch bei $140^{\circ}$ getrocknet noch ca. ${ }^{1 / 8}$ Mol. Wasser und war erst bei $150^{\circ}$ wasserfrei.

Eine Mikroanalyse nach Professor Pregl ergab folgende Resultate :

\section{Elementaranalyse.}

1. Ausgeführt mit Substanz, die bei Atmosphärendruck über Schwefelsüure getrocknet war.

$5,236 \mathrm{mg}$ gaben $0,276 \mathrm{ccm} \mathrm{N}(712 \mathrm{~mm} \mathrm{18,50)}=5,75 \% \mathrm{~N}$

$7,285 \mathrm{mg}$ gaben $2,82 \mathrm{mg} \mathrm{H}_{2} \mathrm{O}=4,33 \% \mathrm{H}$

$$
\begin{aligned}
& 5,33, \mathrm{CO}_{2}=19,96 \% \mathrm{C} \\
& 2,389: \mathrm{CuO}=26,20 \% \mathrm{Cu}
\end{aligned}
$$

Berechnet für

$\mathrm{C}_{4} \mathrm{H}_{3} \mathrm{O}_{4} \mathrm{NCu}+2,7 \mathrm{H}_{3} \mathrm{O}$

$$
\begin{aligned}
& \mathrm{C}=19,73 \\
& H=4,31 \\
& N=5,76 \\
& \mathrm{Cu} \equiv 26,13 \\
& 1,745 \mathrm{mg} \mathrm{H}_{2} \mathrm{O}=3,01 \% \mathrm{H} \\
& 5,67>\mathrm{CO}_{2}=23,82 \% \mathrm{C} \\
& 2,545 \text { > } \mathrm{CuO}=31,33 \% \mathrm{Cu}
\end{aligned}
$$

Gefunden

19,96

26,20

2. $8,567 \mathrm{mg}$ feuchter Substanz verloren beim Trocknen auf $140^{\circ} 2,076 \mathrm{mg}=24,33 \%$. Die so erhaltenen $6,491 \mathrm{mg}$ ergaben

Für $\mathrm{C}_{4} \mathrm{H}_{5} \mathrm{NO}_{4} \mathrm{Cu}+1 / 2 \quad \mathrm{H}_{2} \mathrm{O}$ berechnet sich $23,57 \% \mathrm{C}$, $2,97 \% \mathrm{H}$ und $31,22 \% \mathrm{Cu}$.

3. $4,69 \mathrm{mg}$ der bei $150^{\circ}$ getrockneten Substanz gaben

$$
\begin{array}{cc}
\text { gefunden } & \begin{array}{c}
\text { berechnet für } \\
\mathrm{C}_{4} \mathrm{H}_{6} \mathrm{NO} \mathrm{Cu}
\end{array} \\
1,01 \mathrm{mg} \mathrm{H}_{3} \mathrm{O}=2,41 \% \mathrm{H} & 2,59 \% \mathrm{H} \\
4,25: \mathrm{CO}_{3}=24.72 \% \mathrm{C} & 24,66 \% \mathrm{C} \\
1,888 \% \mathrm{CuO}=32,16 \% \mathrm{Cu} & 32,66 \% \mathrm{Cu}
\end{array}
$$

Wasserbestimmung.

Um die maximale Wassersättigung zu ermitteln, wurde die Substanz erst 30 Minuten in feuchter Atmosphäre unter einer Glasglocke stehen gelassen.

1. 6,65 mg der mit Wasserdampf gesältigten Substanz verloren beim Trocknen im Vakuum über Schwefelsüure in 20 Minuten $1,470 \mathrm{mg}=22,11 \%$ Wasser. 
2. 6,65 mg derselben Substanz verloren beim Trocknen auf gleiche Weise in einer Stunde 1,574 $\mathrm{mg}=23,67 \%$ Wasser.

3. $6,65 \mathrm{mg}$ verloren beim Trocknen auf $150^{\circ}$ bis zur Gewichtskonstan\% $1,960 \mathrm{mg}=29,47 \%$.

Eine Berechnung ergibt, daB das Salz in feuchter Atmosphäre 4,52 Mol. Wasser enthält und daB es im Vakuum über Schwefelsäure getrocknet nach 20 Minulen noch 1,18 Mol., nach 1 Stunde nur noch 0,89 Mol. Wasser enthielt. Beim Stehen über Schwefelsäure unter Atmosphärendruck behielt das Salz ca. 3 Mol., beim Trocknen auf $140^{\circ}$ ca. 1/2 Mol. Wasser.

Die Analysen und auch die Wasserbestimmung stimmen auf asparaginsaures Kupfer. Ritthausen ${ }^{1}$ ) gibt an, daB Kupferasparagat erst bei $150-156^{\circ}$ sein Wasser verliert und daß bei $160^{\circ}$ Zersetzung eintritt. Der Wassergehalt betrage 29,09\%. Dessaignes ${ }^{2}$ ) fand beim Trocknen auf $160^{\circ} 31,78 \%$ Wasser $=5$ Mol. Curtius $\mathrm{u} . \mathrm{Koch}^{3}$ ) geben an, daß es nach dem Trocknen über Schwefelsäure $3 \mathrm{Mol}$. Wasser enthält. Nach Hofmeister ${ }^{4}$ ) ist es leicht löslich in verdünnter kochender Essigsäure. Abderhalden u. Kautzsch ${ }^{5}$ ) beschrieben ein asparaginsaures Kupfer, welches von den darin enthaltenen 4,5 Mol. $\mathrm{H}_{2} \mathrm{O}$ im Vakuumexsikkator sehr langsam $3 \mathrm{Mol}$., bei $110-120^{\circ}$ ein weiteres Mol., den Rest von 0,5 Mol. erst bei $150^{\circ}$ abgab.

Der Ester, in welchem die Aminosäure sich vorfand, destillierte unter $16-19 \mathrm{~mm}$ Druck bei $102^{\circ}$ über, während derjenige der Asparaginsäure nach E. Fischer ${ }^{6}$ ) unter $11 \mathrm{~mm}$ Druck einen Siedepunkt von $126,5^{\circ}$ hat.

Nach Hofmeister (a. a. 0.) löst sich Kupferasparagat in 234 Teilen kochendem und in 2870 Teilen kaltem Wasser, während das von mir erhaltene Salz eine vièl geringere Löslichkeitsdifferenz aufwies. Es löste sich viel schwerer in heißem

1) Journ. f. prakt. Chem., Bd. 107, S. 229 (1869).

2) Gmelin, Handb. Supl. II, S. 897.

3) Journ. f. prakt. Ghem., Bd. 146, S. 486 (1888).

4) Ann. Bd. 189, S. 20 (1877).

5) Diese Zeitschr., Bd. 64, S. 459 (1910).

$\left.{ }^{6}\right)$ Berichte Bd. 34, S. 453 (1901). 
Wasser und fiel aus der erkalteten Lösung erst nach dem Verdunsten eines Teiles des Wassers und nach längerem Stehen aus. Es war auch entgegen dem Befund desselben Forschers in kochender verdünnter Essigsäure kaum löslich.

Die freie Säure war in Wasser nicht sehr schwer löslich. Curtius u. Koch (a. a. O.) erhielten aus einem Salz, welches sie als Kupferasparagat ansprachen, durch Zersetzen mit Schwefelwasserstoff eine Aminosäure. Diese schien sich im Wasser viel leichter zu lösen als die aus Asparagin dargestellte Asparaginsäure, vielleicht hatten sie dieselbe Substanz in Händen, die ich hier bei der Hydrolyse des alkalilöslichen Eiweiß aus Mais erhalten habe.

Aus der 3. und 5. Krystallfraktion konnte kein Kupfersalz, welches dem obigen glich, gewonnen werden. Durch einen Zufall war also diese Aminosäure nur in die eine Krystallfraktion gelangt, welche auch nur geringe Spuren eines anderen Kupfersalzes gab.

Bestimmung der Basen im Eiweif.

Die Hydrolyse und Bestimmung der Basen wurde genau nach der Vorschrift von $F$. Wei ${ }^{1}$ ) ausgeführt.

$49,71 \mathrm{~g}$ Eiweiß $=44,60 \mathrm{~g}$ Trockensubstanz wurden mit $60 \%$ iger Schwefelsäure digeriert, hierauf mit Wasser auf $30 \%$ verdünnt, durch 6 stündiges Kochen am Rückflußkühler hydrolysiert. Nach dem Abkühlen mit Wasser verdünnt und filtriert, wobei $6,60 \mathrm{~g}=14,78 \%$ Rückstand verblieb. Bei der Hydrolyse mit Salzsäure betrug der Rückstand ca. $28 \%$.

Das Resultat war folgendes:

Das untersuchte alkalilösliche Eiweiß enthielt 10,50\% Gesamtstickstoff, welcher sich wie folgt verteilt :

$\begin{array}{lll}\text { Hydrolysenrückstand } 0,59 \% \mathrm{~N} & \\ \text { Huminstickstoff I } & 0,59 \% & \\ \text { Ammoniatstickstoff } & 0,51 & =0,62 \% \text { Ammoniak } \\ \text { Histidinstickstoff } & 0,92 & \equiv 3,49 \% \text { Histidin } \\ \text { ArgininstickstoIf } & 1,58 & =4,92 \% \text { Arginin } \\ \text { Cysinstickstoff } & 0,65 & =3,89 \% \text { Lysin } \\ \text { Aminoszuren-N } & 4,99 \% . & \end{array}$

- J) Diese Zeitschr. Bd. 52, S. 108 (1907).

Hoppo-ssglere Zritertrift r. phrainl. Chemie. XCV. 
Osborne und Clapp hatten für ihr alkalilösliches Protein aus Maissamen mehr Arginin (7,06\%) und Ammoniak (2,12\%), dagegen weniger Lysin $(2,93 \%)$ und Histidin $(3,00 \%)$ gefunden. Die Basen Histidin, Arginin, Lysin wurden als Pikrolonate bezw. Pikrate isoliert und charakterisiert.

\section{H. Autolysenversuche.}

Aus den Embryonen konnten basische Produkte, wie solche sich in Weizenembryonen vorfinden, nicht isoliert werden, dagegen fand sich der gröBte Teil des Stickstoffs in Form von Eiweißkörpern vor. Es wurde daher untersucht, ob bei einer Autolyse sich basische Körper aus dem Eiweiß abspalten. Zur vorläufigen Orientierung wurden nur kleine Mengen autolysiert und die Autolysenflüssigkeit der Analyse unterworfen.

Autolyse mit Material A.

$24,8 \mathrm{~g}$ Trockensubstanz wurden mit $150 \mathrm{ccm}$ Leitungswasser, 2,8 $\mathrm{g} \mathrm{NaF}$ und etwas Chloroform unter Xylol 16 Tage bei $37-40^{\circ}$ stehen gelassen, hierauf wurde abgesaugt, der Rückstand wiederholt gewaschen, das etwas trübe Filtrat von Xylol und Chloroform befreit und auf $500 \mathrm{ccm}$ aufgefültt. Das Filtrat hatte starken Geruch nach Benzaldehyd.

a) Stickstoffbestimmungen ergaben folgende Resultate:

$\begin{array}{cllr}\text { fällbar durch Kupferhydroxyd } & 0,035 \% \mathrm{~N}=10,1 \% & \begin{array}{r}\text { vom gesamten } \\ \text { wasserlösl. }\end{array} \\ \text {, } & 0,059 & 17,1 \% \\ \text {, Bleiessig } & \text { Phosphorwolframsäure } & 0,014 & 4,1 \% \\ \text { wasserlöslicher Gesamt-N } & 0,237 \% \mathrm{~N} & 68,7 \% \\ & 0,344 \% . & \end{array}$

Das mit Äther extrahierte Material A hatte ergeben:

1. fällbar mit Phosphorwolframsäure $0,32 \% \mathrm{~N}$

2. Aminosäuren usw. $0,46 \% \mathrm{~N}$.

Dies gibt berechnet auf unextrahiertes Ausgangsmaterial A

1. $0,147 \% \mathrm{~N}$ ). während das der Autolyse ( $1.0,073 \% \mathrm{~N}$.

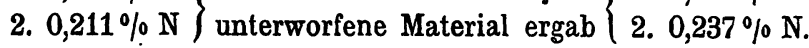

Überblickt man diese Resultate, so èrgibt sich, daß die untersuchten Embryonen keine eiweißspaltenden Fermente enthielten. 
b) Zuckerbestimmung im wässerigen Extrakt der Autolyse.

Diese wurde nach der Methode von Bertrand ausgeführt. Man filtriert das durch Reduktion der Fehlingschen Lösung entstandene Kupferoxydul ab, löst es in Ferrisulfatlösung und titriert das gebildete Ferrosalz mittels Kaliumpermanganat.

$1 \mathrm{ccm}$ der verwendeten Kaliumpermanganatlösung entsprach dabei $9,834 \mathrm{mg} \mathrm{Cu}$.

1. $25 \mathrm{ccm}$ der Lösung $=1,24 \mathrm{~g}$ Substanz verbrauchten $5,60 \mathrm{ccm} \mathrm{KMnO}{ }_{4}=55,1 \mathrm{mg} \mathrm{Cu}=27,9 \mathrm{mg}$ oder $2,25 \%$ als Glukose berechnet.

2. $25 \mathrm{ccm}$ Lösung wie oben behandelt braucht $5,8 \mathrm{~cm}$ $\mathrm{KMnO}_{\mathrm{s}}=57,0 \mathrm{mg} \mathrm{Cu}=28,9 \mathrm{mg}$ oder $2,33 \%$ Glukose.

Mittel: 2,29\% als Glukose berechnet (event. Maltose enthaltend).

\section{Polysaccharide.}

Je $25 \mathrm{ccm}$ Lösung wurden mit $4 \mathrm{ccm}$ konzentrierter Salzsäure gekocht, hierauf weiter wie oben behandelt.

1. verbrauchte $7,5 \mathrm{ccm} \mathrm{KMnO}_{4}=73,8 \mathrm{mg} \mathrm{Cu}=38 \mathrm{mg}$ oder $3,06 \%$ Invertzucker.

2. verbrauchte $7, \tilde{5} \mathrm{ccm} \mathrm{KMnO}_{4}=3,06 \%$.

Hittel: $3,06 \%$ Gesamtzucker, folglich $3,06-2,29=0,77 \%$ Pol y sacch aride (ber. als Glukose).

Da weder der alkoholische noch der wässerige Extrakt der Maiskeime nennenswerte Mengen von Kohlenhydraten ergeben hatten, so scheinen diese in Form von unlöslichen Verbindungen darin vorzukommen und erst durch ein vorhandenes Enzym, welches bei der Autolyse wirkte, in wasserlösliche Form überzugehen.

$$
\text { c) Prüfung auf Glykoside. }
$$

Da sowohl bei der Untersuchung des Alkolsolextraktes als auch bei einem der Autolysenversuche intensiver Geruch nach Benzaldehyd aufgetreten war, so lag die Vermutung nahe, daß in den Embryonen des Mais ein Glykosid vorhanden 
sei. In den Gramineen, Panicum und Sorghum z. B. kommt das Glykosid Dhurrin $\mathrm{C}_{6} \mathrm{H}_{6} \mathrm{C}-\mathrm{CH}_{-} \mathrm{H}_{6} \mathrm{H}_{11} \mathrm{O}_{4}$ vor. Trotz mehrfacher Versuche ist es nicht gelungen, den Benzaldehyd durch sein Hydrazon zu charakterisieren und HCN nachzuweisen.

\section{d) Pentose bei der Autolyse.}

In einem letzten Versuche wurden von den nicht ausgelesenen Embryonen B $500 \mathrm{~g}=474,8 \mathrm{~g}$ Trockensubstanz gemahlen und mit $1900 \mathrm{~g}$ Leitungswasser bei $37-38^{\circ}$ der Autolyse überlassen, wobei am Anfang starke Gasentwicklung auftrat. Nach 2 Wochen wurde der Rückstand abgepreßt, mit Wasser gründlich gewaschen und vom Waschwasser abgepreßt. Das Filtrat der ersten Abpressung betrug $1000 \mathrm{ccm}$; es gab auch nach längerer Zeit keine Reaktion auf Blausäure, dagegen gab eine Probe davon eine intensive Reaktion auf Pentose.

Die Hälfte des Filtrates wurde der Furfuroldestillation nach Tollens unterworfen, jedoch mit der kleinen Modifikation, daß am Anfang mit Dampf ohne Zugabe von Salzsäure destilliert wurde. Die Autolysenflüssigkeit gab 0,85\% Pentose, bestimmt als Phloroglucid. Diese Pentosenmengen dürften wohl zum größten Teil aus Pentosiden abgespalten sein.

\section{J. Darstellung organischer Basen.}

Trotz vieler umständlicher Versuche wurden aus einer großen Quantität von entfettetem Material keine der bekannten Basen erhalten. Die Aufarbeitung des wässerigen oder alkoholischen Auszuges geschah nach den üblichen Methoden. Es wurden wohl bei der Trennung der Basen mit Silbernitrat und Baryt Fällungen bei den einzelnen Fraktionen erhalten, aber es konnte nur in einem Falle eine krystallisierende Substanz erhalten werden. Es gelang, aus der Argininfraktion nur Guanidin und eine merkwürdige Substanz darzustellen.

Beim Verarbeiten von ca. 11 Kilo entfetteten Materials wurde eine größere Menge einer Argininfraktion erhalten, welche eine eigenartige Gallerte darstellte, die zu einer glasigen 
Masse eintrocknete. Diese Substanz gab die Biuretreaktion; mit Diazobenzolsulfosäure starke Rotfärbung, mit Brückes Reagens weiße Trübung. Aus der wässerigen Lösung wurden zunächst 3 Fraktionen hergestellt.

I. Fraktion gab mit Natriumpikrat eine Ausscheidung von gelben Krystallen (tonnenförmige, zu Sternen vereinigte Sechsecke). Das umkrystallisierte Pikrat gab Krystalle von hackenförmiger Gestalt, es bräunte sich bei $190^{\circ}$, schäumte bei $226^{\circ}$ und schmolz noch nicht bei $250^{\circ}$. Es lieferte beim Zersetzen mit Salzsäure 77,96\% Pikrinsäure, woraus sich für die darin enthaltene Base ein Molekulargewicht von 65 berechnet.

Die salzsaure Lösung gab mit Neßlers Reagens eine rein weiße Fällung. Das Golddoppelsalz enthielt 49,41\% $\mathrm{Au}$ und $35,06 \%$ Cl. Für Guanidingoldchlorid berechnet sich aus der Formel $\mathrm{CH}_{3} \mathrm{~N}_{3} \mathrm{HAuCl}_{4} 49,40 \%$ Au und $35,53 \% \mathrm{Cl}$.

II. Fraktion. Die Mutterlauge des ersten Pikrats wurde mit mehr Natriumpikratlösung versetzt und gab nach einiger Zeit $0,97 \mathrm{~g}$ gelbe Krystalle, welche im Wasser leichter löslich waren als diejenigen der I. Fraktion. Beim Umkrystallisieren aus Wasser verblieben $0,504 \mathrm{~g}$ des Pikrats, das sich bei $190^{\circ}$ bräunte und bei $250^{\circ}$ noch nicht schmolz. Es wurde mit Salzsäure versetzt und von der Pikrinsäure ausgeäthert. $0,4950 \mathrm{~g}$ gaben $0,4330 \mathrm{~g}=87,52 \%$ Pikrinsäure, was einem Molekulargewicht der Base von 32,7 entspräche. Die von der Pikrinsäure befreite Lösung wurde mit Goldchlorid versetzt, das entstandene Goldsalz enthielt $52,08 \% \mathrm{Au}$.

$$
\begin{aligned}
& 0,1022 \text { g Substanz gab } 0,0537 \mathrm{~g} \mathrm{Au}=62,52 \% \text {, } \\
& 0,0964 \text { g Substanz gab } 0,0510 \mathrm{~g} \mathrm{Au}=51,64 \% \text {. }
\end{aligned}
$$

Das Goldsalz bräunte sich bei $181^{\circ}$, sinterte bei $194^{\circ}$ und schmolz bei $295^{\circ}$ (korr.). Die geringe Menge desselben erlaubte keine Analyse.

III. Fraktion. Die Mutterlauge dieses zweiten Pikrats wurde ungefähr auf die Hälfte eingeengt, mit konzentrierter Natriumpikratlösung versetzt und wie die beiden vorhergehenden Fraktionen behandelt. Es resultierten $1,025 \mathrm{~g}$, nach dem Umkrystallisieren 0,528 g eines Pikrates, welches einen Schmelzpunkt von 215 (korr.) zeigte, wobei es sich kurz vor 
dem Schmelzen bräunte. Das daraus gewonnene Goldchloridsalz hatte einen Schmelzpunkt von $282,5^{\circ}$ (korr.) und einen Goldgehalt von $54,86 \%$.

$0,1637 \mathrm{~g}$ Salz gaben $0,0898 \mathrm{~g} \mathrm{Au}$. Auch von dieser Fraktion war nicht genügend für eine weitere Analyse vorhanden.

IV. Fraktion. Durch weiteres Einengen der Mutterlauge und Versetzen mit viel Natriumpikratlösung wurden $0,961 \mathrm{~g}$ eines grünlichgelben Pikrates erhalten, das nach dem Uimkrystallisieren aus Wasser $0,788 \mathrm{~g}$ gab. Der Schmelzpunkt dieser gereinigten Krystalle lag bei $291^{\circ}$ (korr.), wobei Aufschäumen stattfand.

Eine Elementaranalyse der Substanz und die Prüfung auf Amidstickstoff im Apparat von van Slyke ergab, daß diese Fraktion hauptsächlich aus Ammonpikrat bestand.

Die Mutterlaugen der Pikrate aus den beschriebenen Fraktionen wurden mit Schwefelsäure von der Pikrinsäure befreit und die Lösung mit Phosphorwolframsäure gefällt, wobei ein dicker krystallinischer Brei resultierte. Aus der Fällung wurde die Base in bekannter Weise dargestellt. Diese Lösung gab mit Neßlers weiße Trübung, mit Millons Reagens Fällung, mit Goldchlorid und Cadmiumkaliumjodid Trübung. Die Biuretreaktion war positiv, mit Diazobenzolsulfosäure entstand starke rote Färbung. Die Knoopsche Reaktion mit Brom auf Histidin war negativ; ebenso die Reaktion auf Cytosin. Kaliumpermanganat wurde in schwefelsaurer Lösung momentan entfärbt. Behufs weiterer Charakterisierung wurden nun folgende Versuche angestellt.

Die Lösung wurde mittels Silbersulfat von überschüssigem Baryum und von Chloriden befreit, das Filtrat eingeengt und über Natronkalk evakuiert, wobei ein gelber, klarer Sirup verblieb. Eine Probe desselben gab beim Erhitzen mit Fehling scher Lösung Spuren von Kupfer, die Flüssigkeit färbte sich rotviolett und es trat intensiver Geruch nach Trimethylamin auf. Mit Phenylhydrazin gab eine weitere Probe einen hellgelben Krystallbrei, der bei ca. $20^{\circ}$ erweichte. Ammoniakälische Silbernitratlösung gab eine weiße Fällung (wohl von noch vorhandenen Chloriden), welche sich sofort schwärzte. Phloroglucin mit Salz- 
säure gab beim Erwärmen keine Rotfärbung, wodurch die Anwesenheit eines Pentosids ausgeschlossen ist. Ein Teil des Sirups wurde mit Goldchlorid und Salzsäure versetzt, es entstand eine ölige Ausscheidung, welche sich beim Erwärmen löste, beim langsamen Abkühlen nur Spuren von Krystallen gab.

Der Rest des Sirups wurde eingedunstet und der Krystallisation überlassen. Im Exsikkator über Schwefelsäure verblieb nach längerer Zeit eine amorphe, glasige Masse. Diese wurde mit Wasser aufgenommen und die freie Base darzustellen versucht. Die Lösung wurde rorsichtig mit frisch gefälltem Silberoxyd versetzt, bis eine Probe des Filtrates mit Silbernitrat nur roch schwache Trübung gab. Es ließ sich jedoch ein Überschuß von Silberoxyd nicht vermeiden, nach längerem Stehen gab eine Probe mit wenig Salzsäure eine geringe Fällung. Daher wurde von den ausgefallenen Silbersalzen abfiltriert, in das Filtrat Schwefelwasserstoff eingeleitet und das Filtrat vom Silbersulfid bei $60-80 \mathrm{~mm}$ Druck (bei niedrigerem Druck fand Aufschäumen statt) und $50^{\circ}$ vorsichtig eingeengt. Im Vakuum über Natronkalk getrocknet verblieb wieder ein hellgelber Sirup.

Eine Probe desselben, im Wasser gelöst, gab mit viel Alkohol eine Emulsion, welche sich beim Erwärmen klar löste. Die Lösung hinterließ beim Abkühlen ölige Tropfen. Beim Eindunsten dieser Lösung mit etwas Eisessig verblieb nach längerem Stehen im Exsikkator wiederum eine amorphe Masse. Eine zweite Probe gab bei Neutralisation mit Oxalsäure eine Trübung, wobei die Flüssigkeit aufschäumte. Das Filtrat von dieser Trübung konnte auch nicht zur Krystallisation gebracht werden.

Mit Pikrolonsäure entstanden wenig gelbe Krystalle, welche in feinen Nadeln strahlig angeordnet waren.

Die Base wurde nun mit Schwefelsäure neutralisiert und der Krystallisation überlassen. Es verblieb jedoch wiederum wie bei allen anderen Versuchen zur Darstellung eines Salzes ein Sirup, der im Exsikkator glasig erstarrte. Eine Probe gab im Glührobr starkes Aufschüumen, Geruch nach verbranntem Horn, Ammoniak- und (Nikotin-)Geruch.

Die über Schwefelsüure getrocknete Substanz enthielt $17,73^{\circ}, \mathrm{N}$ und nur $2,91 \% \mathrm{H}_{2} \mathrm{SO}_{4}$. 
Eine Bestimmung des Amidstickstoffs mit dem Apparat von van Slyke ergab 5,86\% N. 0,0927 g gaben:

\begin{tabular}{|c|c|c|}
\hline h $6 \mathrm{Min}$ & & \\
\hline 30 & & \\
\hline $1 \mathrm{St}$ & 6.4 & \\
\hline $2^{3} / 4$, & 7,3 & \\
\hline , 3 & $8,6$. & , \\
\hline 6 & 9,1 & \\
\hline 7 & 9,15 , & \\
\hline
\end{tabular}

Es ist also in der Substanz ein Drittel des gesamten Stickstoffs in Form von Amidstickstoff gebunden.

Eine Mikroanalyse nach Pregl ergab folgende Resultate:

Stickstoffbestimmung:

$6,268 \mathrm{mg}$ gaben bei $712 \mathrm{~mm}$ und $20^{\circ} 1,005 \mathrm{ccm}=17,50 \% \mathrm{~N}$ 3,370 , , , 716 , , $20^{\circ} 0,550,=17,91 \%$,

Bestimmung von Kohlen- und Wasserstoff im Rohr mit Bleichromat:

4,572 $\mathrm{mg}$ gaben $3,215 \mathrm{mg} \mathrm{H}_{2} \mathrm{O}$ und $7,20 \mathrm{mg} \mathrm{CO}$ $=7,87 \% \mathrm{H}$ und $42,95 \% \mathrm{C}$.

Die obigen Bestimmungen sowie auch die folgenden des Wassers wurden mit der über Schwefelsäure getrockneten Substanz ausgeführt.

Wasserbestimmung :

12,790 mg der Substanz verloren beim langsamen Erhitzen: in $25 \mathrm{Min}$. bis $104^{\circ} 0,840 \mathrm{mg}=6.36 \% \mathrm{H}_{2} \mathrm{O}$

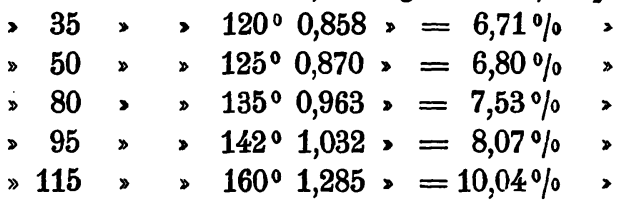

Bei ca. $145^{\circ}$ fand ein Aufblähen der Substanz statt.

Für die freie Base berechnet sich aus der obigen Analyse (nach Abzug der in der Substanz enthaltenen 2,94\% $\mathrm{H}_{2} \mathrm{SO}_{4}$ ) folgende Formel: $\mathrm{C}_{17} \mathrm{H}_{37} \mathrm{~N}_{6} \mathrm{O}_{8}$, oder $\mathrm{C}_{17} \mathrm{H}_{32} \mathrm{~N}_{6} \mathrm{O}_{6}+2,5 \mathrm{H}_{2} \mathrm{O}$. Molekulargewicht 461,4 , davon ca. $10 \%=2,5$ Mol. Wasser.

$\begin{array}{lr}\text { Berechnet für } & \text { Gefunden } \\ \mathrm{C}_{17} \mathrm{H}_{37} \mathrm{~N}_{6} \mathrm{O}_{8}, 5 & \\ \mathrm{C}=44,22 \% & 44,25 \% \\ \mathrm{H}=8,08 \% & 805 \% \\ \mathrm{~N}=18,22 \% & 18,23 \%\end{array}$


Wenn man nur 3 Atome Stickstoff in der Substanz annimmt, so gibt dies für Kohlenstoff 8,5 Atome; die Formel mit 8 Atomen gibt für $\mathrm{C}$ viel $\mathrm{zu}$ kleine, diejenige mit 9 Atomen zu große Werte.

Über die Natur dieses Körpers kann leider nichts Positives gesagt werden. Es steht nur fest, daß von je 3 Stickstoffatomen eines in Form von Amidstickstoff darin vorkommt. Bei der wahrscheinlicheren und aus der Formel sich ergebenden Annahme von 6 Stickstoffatomen sind auch 2 Aminogruppen anzunehmen, von denen nur eine in $\alpha$-Stellung zur Carboxylgruppe stehen könnte, da die Reaktion mit salpetriger Säure erst nach 7 Stunden quantitativ verlaufen war. Jedenfalls handelt es sich um keine der bekannten Aminosäuren.

Trotzdem es nicht gelungen war, aus der zuletzt beschriebenen Substanz ein krystallisierendes Derivat zu erhalten, so ist doch kaum anzunehmen, da $\beta$ bei den Prozeduren der wiederholten Reinigungen noch ein kompliziertes Gemisch verschiedener Substanzen darin enthalten sei, zudem ja auch aus der Argininfraktion einige Pikrate, darunter das Guanidinpikrat, abgeschieden werden konnten.

Auf Grund der Beobachtungen scheint die Annahme berechtigt, daß es sich um ein Purinderivat mit 2 Aminogruppen handeln kann. In der Argininfraktion sind ja schon wiederholt Purinkörper aufgefunden worden, da die Trennung mit Silbersalzen nicht ganz quantitativ ist.

Zusammenfassung der Ergebnisse.

Aus den im vorigen mitgeteilten Versuchsergebnissen lassen sich folgende Sätze aufstellen:

Die Zusammensetzung der Maiskeime weicht in vieler Beziehung von derjenigen der in unserm Laboratorium eingehend untersuchten Weizenkeime verschiedener Provenienz ab. Diese $A b$ wejchung besteht in erster Linie darin, daß in den Weizenkeimen verschiedene krystallisierbare Stickstoffverbindungen (EiweiBabbauprodukte) in nicht unbeträchtlicher Menge enthalten sind, welche in den Maiskeimen nicht aufgefunden werden konnten. In den zwei verschiedenen unter- 
suchten Sorten von Maiskeimen konnte kein Arginin und nur höchstens Spuren von Glutamin nachgewiesen werden. Überraschend war das Vorhandensein von Guanidin, eine Verbindung, die in Weizenembryonen nicht aufgefunden wurde. Daneben wurde in der gleichen Fraktion eine Base unbekannter Konstitution aufgefunden. Bekanntlich enthalten die Malzkeime eine Base, das Hordenin = Paraoxyphenyläthyldimethylamin. Die aufgefundene Base ist kein Hordenin. Man darf wohl annehmen, daß die den Maiskeimen zugeführten Bausteine des Eiweißmoleküls nahezu vollständig zum Aufbau des Eiweißes verbraucht werden, in den Weizenkeimen dagegen eine partielle Anhäufung dieser Produkte erfolgt. $\mathrm{Ob}$ das Guanidin als Zwischenstufe für die Eiweißsynthese in Betracht kommt, erscheint fraglich. Es ist ferner beachtenswert, daß bei der Autolyse der Maiskeime in vitro nur eine geringfügige Aufspaltung von Eiweiß erfolgt. $\mathrm{DaB}$ in der Tat die Quantität nicht-eiweißartiger Stickstoffverbindungen sehr gering ist, wird durch das Ergebnis der quantitativen Untersuchungen noch gestützt.

Der Stickstoffgehalt ist wesentlich niedriger als bei Weizenembryonen; dementsprechend wurde auch der Eiweißgehalt niedriger gefunden. Bei der Hydrolyse des Eiweißes wurden die bekannten Bausteine erhalten, daneben eine Asparaginsäure von abweichenden Eigenschaften.

Auch die Maiskeime enthalten eine größere Menge wasserlöslicher Eiweißkörper sowie eine kleine Menge Globuline; eine Nucleinsäure konnte nicht isoliert werden, aus Weizenembryonen gelingt die Darstellung leicht.

Der Fettgehalt ist ungefähr viermal so groß als derjenige der Weizenkeime. Das Fett enthält Glyceride fester und flüssiger Fettsäuren, daneben Sitosterin und Phosphatide.

Vermutlich kommt in den Maiskeimen ein Glykosid vor; es konnte Pentose nachgewiesen werden, welche bei der Autolyse wohl aus einem Pentosid abgespalten worden ist.

Die Maiskeime besitzen einen ansehñlichen Gehalt an Inositphosphorsäure. 